\title{
Machine perfusion strategies in liver transplantation
}

\author{
Andrea Schlegel ${ }^{1,2,3}$, Xavier Muller $^{3}$, Philipp Dutkowski ${ }^{3}$ \\ ${ }^{1}$ Liver Unit, Queen Elizabeth Hospital, University Hospitals Birmingham NHS Foundation Trust, Birmingham, UK; ${ }^{2}$ Centre for Liver and \\ Gastrointestinal Research, Institute of Immunology and Immunotherapy, College of Medical and Dental Sciences, University of Birmingham, \\ Birmingham, UK; ${ }^{3}$ Department of Surgery and Transplantation, University Hospital Zurich, Zurich, Switzerland \\ Contributions: (I) Conception and design: A Schlegel, P Dutkowski; (II) Administrative support: All authors; (III) Provision of study materials \\ or patients: All authors; (IV) Collection and assembly of data: All authors; (V) Data analysis and interpretation: A Schlegel, P Dutkowski; (VI) \\ Manuscript writing: All authors; (VII) Final approval of manuscript: All authors. \\ Correspondence to: Philipp Dutkowski, MD. Department of Surgery and Transplantation, University Hospital Zurich, Raemistrasse 100, CH-8091 \\ Zurich, Switzerland. Email: philipp.dutkowski@usz.ch.
}

\begin{abstract}
Machine perfusion is a hot topic in liver transplantation and several new perfusion concepts are currently developed. Prior to introduction into routine clinical practice, however, such perfusion approaches need to demonstrate their impact on liver function, post-transplant complications, utilization rates of high-risk organs, and cost benefits. Therefore, based on results of experimental and clinical studies, the community has to recognize the limitations of this technology. In this review, we summarize current perfusion concepts and differences between protective mechanisms of $e x$ - and in-situ perfusion techniques. Next, we discuss which graft types may benefit most from perfusion techniques, and highlight the current understanding of liver viability testing. Finally, we present results from recent clinical trials involving machine liver perfusion, and analyze the value of different outcome parameters, currently used as endpoints for randomized controlled trials in the field.
\end{abstract}

Keywords: Machine perfusion; clinical trials; viability assessment

Submitted Dec 17, 2018. Accepted for publication Apr 09, 2019.

doi: $10.21037 / \mathrm{hbsn} .2019 .04 .04$

View this article at: http://dx.doi.org/10.21037/hbsn.2019.04.04

\section{Introduction}

It has been an ongoing and fascinating dream for more than 100 years to keep organs alive outside of the human body (1). However, only relatively small steps have been made to achieve this prestigious goal, despite numerous technical advances in the field of organ perfusion, including sophisticated pump heads, shielded tubes, filter developments, blood based perfusates, new oxygen carriers or computerized fully automatic circuit controls (2). Of note, while the process of organ donation, cold storage and transport appears completely reversible in healthy livers upon implantation in-vivo, with full function for many years, explanted organs, even of "good quality", experience severe injury during only a few hours or days of cold storage or ex-situ perfusion (3). The reason behind remains the poorly understood metabolic needs, that determine pathways of injury or repair after cold or warm ischemia (4). Even more challenging is the task, therefore, to improve or repair injured organs ex-situ (Figure 1), a claim, which has however been frequently used by several investigators, in face of an ongoing organ shortage (5-8).

In this review we specifically aim to describe limitations of ex-situ repair of marginal livers by different perfusion techniques. We summarize the current understanding of underlying mechanisms of different perfusion approaches. Next, we highlight recent achievements through clinical trials in the field, and discuss viability assessments. Finally, we suggest the potential design of upcoming trials based on clinical needs.

\section{What are the main machine liver perfusion concepts?}

Two main $e x$-situ perfusion approaches for livers have 


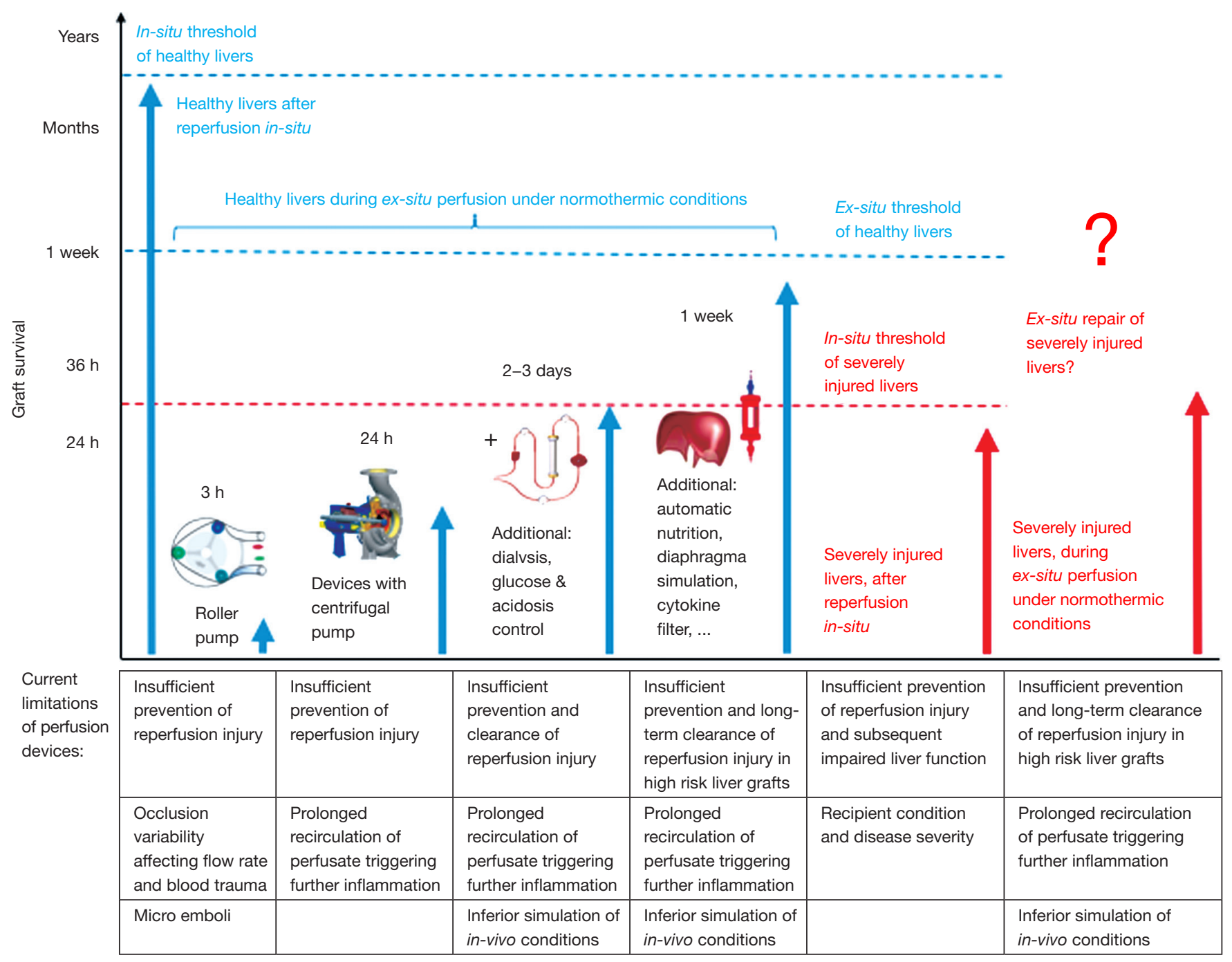

Figure 1 Survival of liver grafts after transplantation in-situ and ex-situ under the best possible normothermic conditions on different devices. Despite recent improvements of machine perfusion technology, liver graft survival during ex-situ perfusion remains limited and strongly depends on the perfusion device and the creation of a near physiological environment. Sophisticated and automatic devices, which include centrifugal pumps, oxygenators with a prolonged capability, dialysis, nutrition and diaphragm simulation may potentially prolong ex-situ survival of healthy livers for up to several day or weeks. Importantly, all devices fail to prevent the initiation of reperfusion injury and also the clearance, which lead therefore to an ongoing inflammation during prolonged perfusion of injured or high-risk liver grafts.

been recently introduced in the clinic, which differ fundamentally in terms of their logistics, and also in terms of their protective mechanism. First, an upfront machine perfusion, immediately after standard procurement, aims to replace the conventional cold storage ("icebox") (Figures 1,2) (6). For this purpose, the organ is placed after procurement on a transportable device, and undergoes continuous perfusion until implantation in the recipient centre $(2,6)$. Sophisticated and expensive systems are used for this approach, mostly at normothermic (NMP) or subnormothermic (SMP) temperatures, with a blood-based perfusate $\left(\right.$ Organox $^{\circledR}$, Transmedics ${ }^{\circledR}$, Liver Assist $\left.{ }^{\circledR}\right)(2,6,9-12)$. A modification of this technique involves an even earlier start of machine liver perfusion already in the donor, e.g., normothermic regional perfusion (NRP), instead of the routine cold in-situ organ flush (Figure 2) $(13,14)$. Following donor cannulation after cardiac arrest and stand off period, NRP is an immediate in-situ perfusion of abdominal organs 


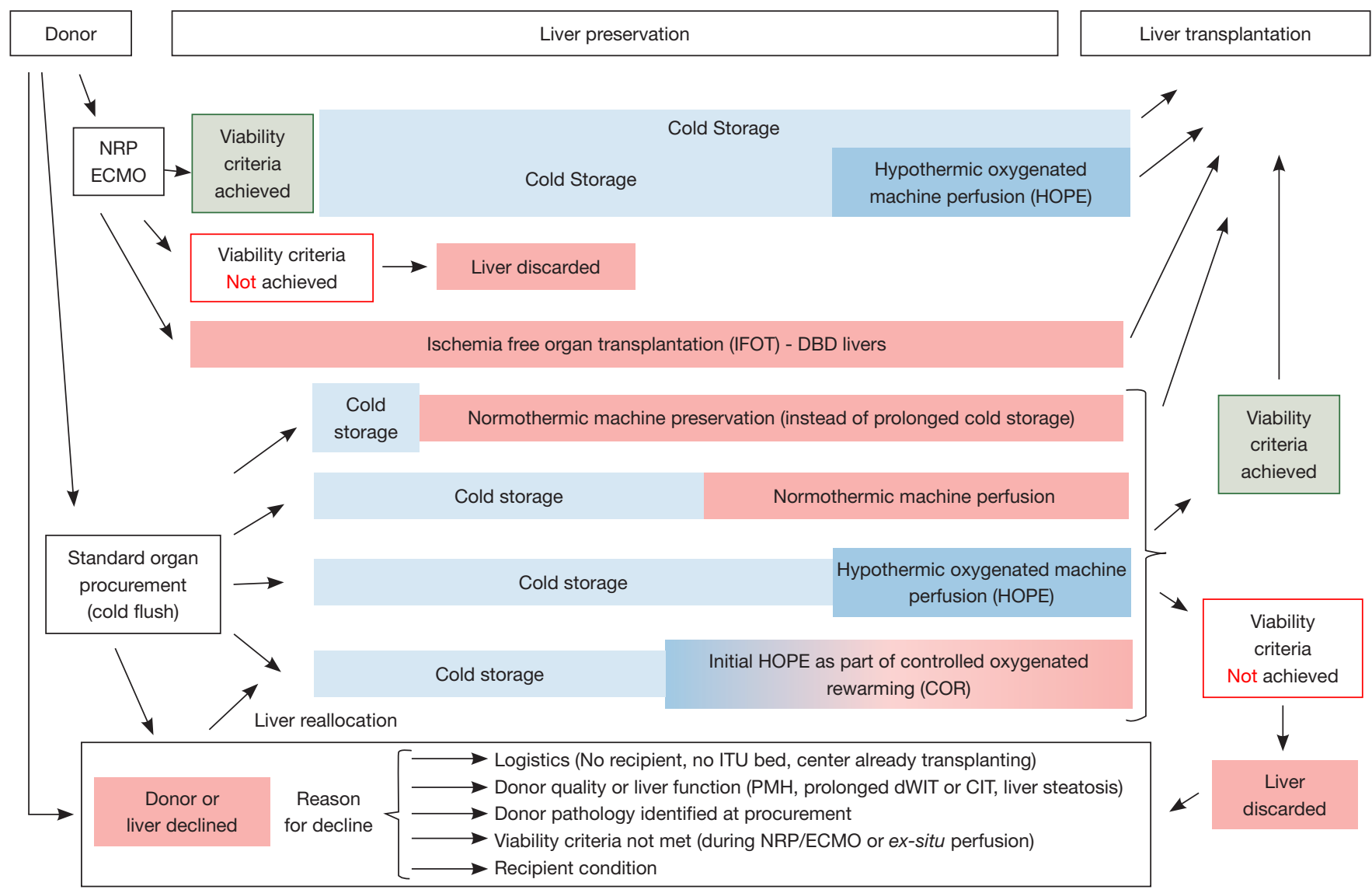

Figure 2 Currently available preservation technology for $i n$-situ and $e x$-situ machine liver perfusion. Multiple new technologies for liver graft treatment and assessment are currently used and tested. Two main concepts include first the replacement of cold storage by perfusion or and endischemic approach, where livers are perfused after cold storage. Different temperatures and perfusate compositions are in use and one main interest is the definition of clinically relevant viability criteria, to decide if an injured or suboptimal liver can be utilized for transplantation or not. Importantly, multiple reasons apply for the decline of a certain graft at different categories and timepoints between the first donor allocation, liver procurement and implantation at the recipient centre. Clinical trials and case series, which are designed to assess the impact of machine perfusion technology should report such reasons and also the risk factors for livers of the declined cohort.

with donor blood for 2-4 hours, where the decision to procure the liver is based on liver enzyme release and lactate values during NRP. In Spain, donor cannulation prior to treatment withdrawal is accepted by law and national data from Maastricht type III donor liver transplantations have therefore shown a very short donor warm ischemia time compared to data provided by other countries $(13,14)$. A logical extreme would be the combination of NRP and NMP, in order to keep the perfused organ without any intermittent cooling and therefore preventing interruption of normothermic perfusion until implantation. This concept leads to a complete abundance of cold ischemia and has been introduced as "ischemia free organ transplantation" in a few human livers (IFOT) (15). Although such procedure avoids repeated temperature changes during liver preservation, the massive technical complexity appears as clear hurdle for a broad clinical introduction (Figure 2). Additionally, the IFOT technique should be first compared to other perfusion techniques.

The alternative machine liver perfusion approach is applied end-ischemically after initial cold storage and liver transport to the recipient center ("repair centers") (16-18). Subsequently, the organs are perfused mostly for a relatively short period prior to implantation. Such end-ischemic perfusion techniques have been applied at all different temperatures, including normothermic and hypothermic or 


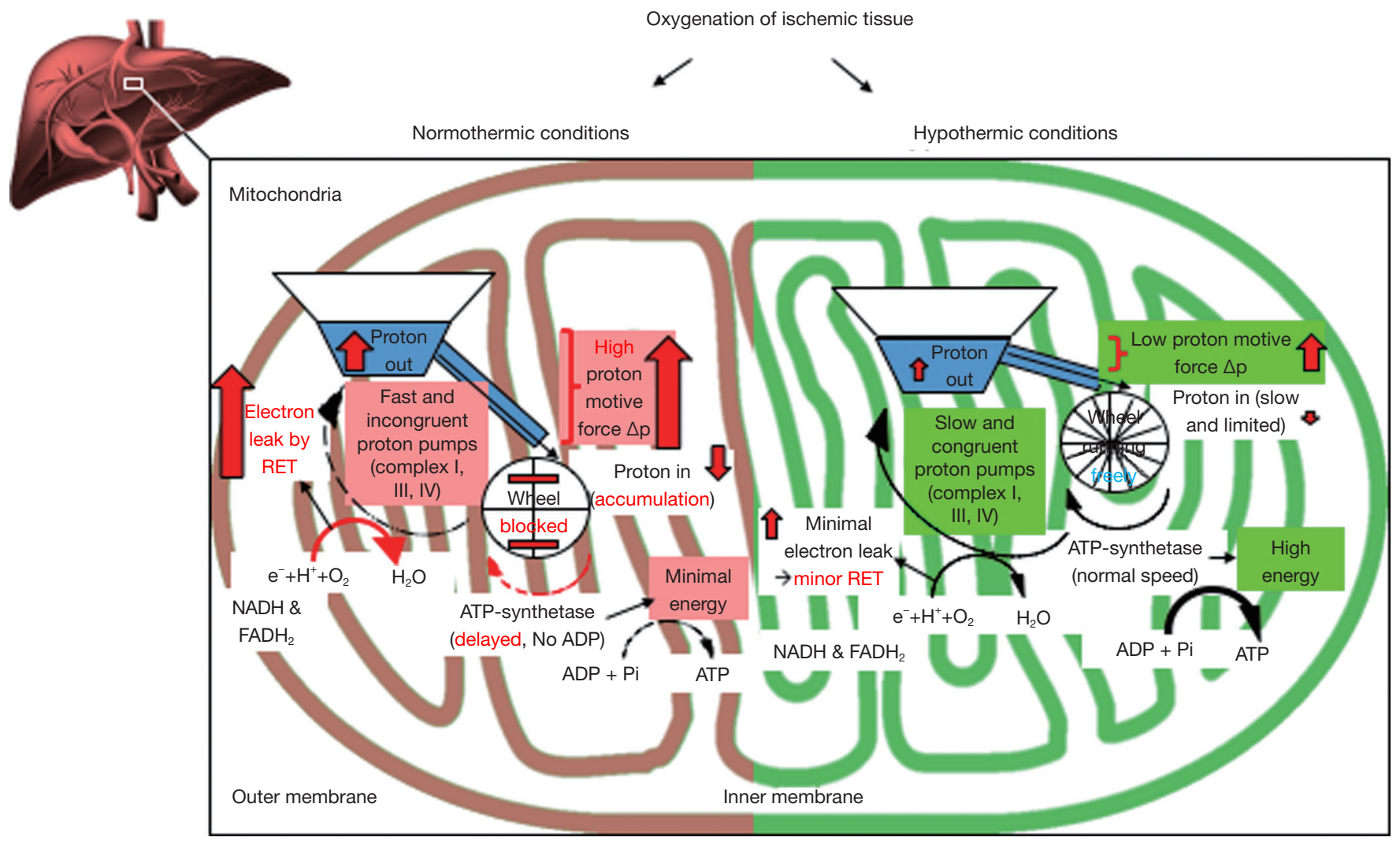

Figure 3 Mechanism of reoxygenation of ischemic liver tissue at different temperatures. Ischemic cells, experience a rapid loss of energy, and most adenosine triphosphate (ATP)_dependent processes are therefore on hold. This phenomenon is paralleled by a significant accumulation of $\mathrm{NADH}$, citric acid cycle- and purine-metabolites, mainly succinate, hypoxanthine, and xanthine. Upon normothermic liver reperfusion, accumulated electron donors, such as NADH and succinate, deliver high amounts of electrons to mitochondrial complex I and II, while ADP is not yet available for the ATP synthetase (complex V), due to previous nucleotide breakdown during ischemia ("wheel is blocked"). This results in an intermittent blockage of proton back flow through the inner mitochondrial membrane, with consecutive high proton motive force, and subsequently reverse electron transfer (RET) between complex II and I, leading to the release of reactive oxygen species (ROS) - from complex I, which occurs within the first few minutes after normothermic reoxygenation. Further parameters, including DAMPs and different cytokines, which are used for viability assessment, appear just downstream. To minimize upfront mitochondrial injury during reoxygenation, reintroduction of oxygen at temperatures below the Arrhenius breakpoint temperature of $15{ }^{\circ} \mathrm{C}$ is required. Subsequently, the reactivity of mitochondrial transfer processes is significantly different and appears comparable to hibernating animals or plants. Mitochondria work more effectively at hypothermic temperatures and upload cellular ATP (Complex V; "wheel works properly"), while consuming cell processes are shut down. Hypothermic oxygenated perfusion (HOPE) after ischemia enables slower but congruent proton pumping through complex I, III and IV and protects therefore, first, from significant mitochondrial ROS-release from complex I, and secondly provides uploaded cellular energy reserves prior to organ rewarming and implantation.

by a combination of both, defined as controlled oxygenated rewarming (COR) (19-22). Although, these techniques are logistically easier and cheaper, because a device transport is not necessary, the initial period of cold ischemia induces severe metabolic depletion before perfusion is started, particularly in high-risk grafts, such as steatotic or donation after circulatory death (DCD) livers (Figures 2,3) (23).
Besides the timing of machine perfusion, the perfusate composition varies substantially among techniques at all temperatures $(9,24)$. While normothermic or subnormothermic perfusions require the presence of red blood cells or artificial oxygen carriers, cold perfusion technologies rely on the amount of dissolved oxygen in the perfusate (9). Hypothermic oxygenated perfusion 
(HOPE) at temperatures between 8-12 degrees is therefore performed with high perfusate oxygen concentrations $(>80 \mathrm{kPa})(4,25)$. Of note, any machine liver perfusion in the cold need strict avoidance of too high perfusion pressures to prevent endothelial sheer stress with subsequent endothelial injury (25).

\section{What is the potential mechanism of protection of liver perfusion approaches?}

The biochemical advantage of normothermic perfusion is the near physiological condition with the option to analyze and treat livers during ex-situ perfusion (23). The disadvantage, however, relies in the fact, that any reperfusion injury is also occurring during normothermic perfusion, as seen in-vivo, even in the absence of leukocytes or platelets, and also if filters for cytokines or danger associated molecular patterns (DAMPs) are used $(23,26)$. This is based on recent evidence that mitochondrial injury is initiated by exposure of hypoxic tissues to oxygen before activation of non-parenchymal liver cells (Figure 3) $(27,28)$. The basic steps of reperfusion injury are consecutively summarized as follows: ischemic cells, regardless of the organ type, experience a rapid loss of energy, and most adenosine triphosphate (ATP)—dependent processes are subsequently on hold $(29,30)$. This phenomenon is paralleled by a massive accumulation of NADH, citric acid cycle- and purine-metabolites, mainly succinate, hypoxanthine, and xanthine (31-33). Upon normothermic liver reperfusion, accumulated electron donors, such as $\mathrm{NADH}$ and succinate, deliver high amounts of electrons to mitochondrial complex I and II, while ADP is not yet available for the ATP synthetase (complex V), due to previous nucleotide breakdown during ischemia $(31,34)$. This results in an intermittent blockage of proton back flow through the inner mitochondrial membrane, with consecutive high proton motive force, and subsequently reverse electron transfer (RET) between complex II and I, leading to reactive oxygen species (ROS)-release at complex I $(32,35,36)$. Any machine perfusion with an oxygenated perfusate after ischemia will therefore induce reperfusion injury to some extent. Importantly, mitochondrial ROS-release occurs within the first minutes of reintroduction of oxygen to ischemic tissues, and triggers an opening of the mitochondrial membrane pore with further release of mitochondrial DNA together with other DAMPs and multiple cytokines $(19,35,37)$. Accordingly, the release of signaling proteins has been recently confirmed during endischemic normothermic perfusion of many different organs, including kidneys, lungs and also livers $(3,26,38-41)$. In contrast, a newly recognized but decisive option to minimize upfront mitochondrial injury during reoxygenation, is cooling of mitochondria below the Arrhenius breakpoint temperature of $15{ }^{\circ} \mathrm{C}$ (42-44), thereby inducing significant changes in the reactivity of mitochondrial transfer processes, as seen in hibernating animals or plants $(45,46)$. Likewise and surprisingly, mitochondria work also more effective at hypothermic temperatures in uploading cellular ATP, when consuming processes are shut down (Figure 3) $(3,41,47,48)$. A similar central role of attenuating mitochondria derived oxidative injury is currently also recognized in other biological fields, such as aging and cancer development (49-51). Hypothermic oxygenated perfusion (HOPE) after ischemia protects therefore, first, from significant mitochondrial ROS-release, and secondly provides uploaded cellular energy reserves prior to organ implantation $(3,19,36)$. Both effects depend, however, on the amount of accumulating metabolites during ischemia, which can in principle also lead to an oxidative injury during HOPE. Of note, the changes in mitochondrial metabolism during HOPE are detectable by perfusate analysis during cold perfusion, which will likewise be available as viability parameters in the future (19,52).

The clinical effect of this hypothermic perfusion approach is demonstrated in recent observational studies in Maastricht III DCD livers $(53,54)$ and by a group from Milan in Maastricht II and III DCD livers, where NRP was combined with endischemic HOPE $(55,56)$. These results have been achieved despite the use of extended DCD liver grafts, and are strikingly different from recent outcomes after end-ischemic normothermic perfusion of human livers $(20,23)$.

\section{Which livers would benefit from machine perfusion?}

Despite the current hype for machine perfusion technology, cold storage remains effective for many grafts, with low cost and enormous simplicity (57). The transplant community has therefore to define thresholds for marginality, where perfusion concepts could add a relevant benefit. A huge variability exists however between countries, centres and surgeons how to define marginal, extended or highrisk livers including DCD grafts (58), and guidelines are therefore needed in the future (59). For example, the type 
and duration of donor warm ischemia is inconsistent in almost every transplant centre and country, e.g., defining functional donor warm ischemia (fDWIT) by an oxygen saturation in peripheral donor blood below $<80 \%$ or $70 \%$ or by a decrease of systolic blood pressure or mean arterial pressure (MAP) below 50 or $60 \mathrm{mmHg}$ (60-62). Additionally, some studies rely more on the duration of the agonal phase (between withdrawal of treatment and circulatory arrest) or asystolic phase, than on fDWIT (time between asystole/circulatory death and cold flush or start of NRP) (63). Donor age is another classical risk factor with huge variations of recommended thresholds for acceptance of DCD livers, ranging between 50 and 90 years of age (63-66). The majority of clinicians would agree, that an advanced donor age $>60$ years allocates a DCD graft to the high risk or extended criteria donor (ECD) group. However, recently cohort studies have demonstrated comparable outcomes and suggested, that advanced donor age alone should not exclude utilization of an otherwise good DCD liver $(64,65)$. Further risk factors in combination with donor age are needed to completely characterize the level of risk in a donor liver. To describe the entire picture of marginality or an ECD graft appears therefore rather complex and includes multiple risk factors, their thresholds and combinations with recipient risk (52). In addition to donor age, donor BMI, warm and cold ischemia times, level of macrosteatosis, graft type and viral status were frequently considered to impact on graft quality $(18,59)$. Among clinicians, there is a general clinical consensus not to use a very "risky" livers for a sick recipient, including for example candidates with advanced portal vein thrombosis, listed for retransplantation or with higher MELD scores, which are already admitted or ventilated on intensive care unit (ICU) (62,67-71).

Several risk score models were therefore developed to capture the cumulative risk in donors and recipients, for example by combing cold and warm liver ischemia, donor age, recipient age, recipient MELD, surrogate parameters of hepatic steatosis, and retransplantation $(62,67,72-74)$. Despite well-known shortcomings of a relatively low overall positive predictive value of all score models, such systems help to decide which liver could be accepted for which recipient $(62,75)$. In addition to centre and surgeons experience, the national donation rate per million inhabitants significantly impact on the level of donor risk accepted for transplantation. Countries with lower donation rates are forced to accept higher risk livers. One good example is Switzerland or Italy, where DCD livers with a prolonged fDWIT time (median $>30 \mathrm{~min}$ ) are usually accepted, in context of a high waiting list mortality (76). Interestingly, centres in countries with lower donation rate are usually more interested in underlying mechanisms of preservation technology to finally apply the perfusion approach with the greatest impact on the function of high risk liver grafts $(16,19,25,47,55,77)$.

Another part of the strategy to use risky livers is the selection of a fairly healthy recipient, as for example a transplant candidate with good liver function, but suffering from hepatocellular carcinoma (HCC). These patients have a prolonged waiting time, which depends on the extra point policy of allocation systems (MELD- or UKELD system: United Kingdom model of end stage liver disease) (78). Implantation of injured livers in cancer patients may generate however a higher risk for tumor recurrence due to a more pronounced reperfusion injury (79-82). Although retrospective series showed inconsistent results of tumor recurrence in relatively low risk DCD and DBD transplants $(79,83,84)$, utilization of higher risk DCD livers, may need optimization by machine perfusion, particularly when used for tumor patients to avoid higher HCC recurrence rates in the future.

\section{How good are recent clinical liver perfusion trials?}

The vast majority of clinical studies are retrospective assessments of new preservation techniques demonstrating mainly feasibility. Relevant conclusions are therefore difficult to obtain. While at least five randomized controlled perfusion trials are currently recruiting participants, only one RCT has been completed in 2018 (6,85). In this study, the authors compared normothermic machine liver preservation against conventional cold storage, and chose peak Aspartate-Aminotransferase (AST) as primary endpoint, which is however only a weak parameter of liver injury after transplantation (6). More clinical convincing would be the impact of machine perfusion on posttransplant complications within a reasonable time-period, e.g., 6 months to 1 year, as for example in the two ongoing multicentric randomized machine perfusion trials on hypothermic liver perfusion (HOPE, D-HOPE). Of note, the lack of transparency in reporting of key risk factors makes a real comparison between different transplant centers and new preservation approaches difficult. For example, the reasons for discard of machine perfused livers are frequently subjective and underreported and lead to major selection bias (6). Additionally, parameters of livers, 
declined just prior to $e x$-situ machine perfusion, assuming their quality appears too inferior to achieve a good outcome with normothermic perfusion, are often not described $(21,86)$. Such practice fails to answer the most relevant clinical question, whether certain preservation techniques can increase the utilization rate and provide more livers, previously declined.

Any future clinical trial should base on a uniform policy how to discard livers for transplantation. Unfortunately, most retrospective clinical studies, which aim to assess the impact of NRP or end ischemic NMP, fail to clearly demonstrate, why livers were declined prior to or during perfusion. It is also necessary to report parameters of injury during liver perfusion, similarly as done in kidney and lungs, to quantify the amount of circulating cytokines and DAMPs $(26,39,87)$. In this context, the group of Chris Watson has demonstrated in a very detailed and transparent way, how they use reperfusion injury to assess livers during endischemic NMP (20). These investigators also reported based on which criteria they accepted or declined high-risk livers. In this context, perfusate lactate showed its inferiority to predict liver function and to prevent PNF (20). Additionally, bile flow alone was also a weak predictor of outcomes and complications. However, bile parameters including bicarbonate and $\mathrm{pH}$ at a threshold 7.4 , clearly discriminated livers with and without biliary complications in this series from Cambridge $(20,23)$. Other retrospective case studies, where liver grafts were assessed during endischemic NMP, did not describe the reasons for decline transparently enough (86). Good DBD livers, declined for logistical reasons (Figure 4), perform equally well on any normothermic device with or without previous cold storage $(6,88)$. In contrast, for example DCD livers or steatotic grafts, accumulate further invisible injury during prolonged cold storage, and experience the full reperfusion injury cascade, which suddenly becomes visible end enables quantification during endischemic NMP $(41,89)$. Based on this, the detailed presentation of reasons why livers were declined, in any clinical series is of utmost importance (Figures 2,4).

\section{What is currently known in terms of viability assessment during machine liver perfusion?}

All perfusion techniques have been investigated regarding liver metabolism and release of liver specific enzymes as markers of hepatocellular injury. For example, during NRP or NMP, markers of liver injury are now routinely analyzed and led to a first suggestion of a combination of parameters, which may serve as viability criteria (Figure 2). Yet, most viability markers currently rely rather on measurement of liver injury than on hepatocellular or biliary function. In a recent paper, Watson et al. demonstrated impressively, and very detailed, that such markers appear very insufficient and do not answer the question, if a certain liver is too high risk for transplantation. For example, Lactate clearance requires very few hepatocytes only and when a liver demonstrates lactate clearance, it does not automatically mean, that this graft will work properly in the recipient and moreover protect from ITBL.

Some authors have therefore suggested to assess also bile fluid, not only in terms of quantity, but also the composition. And a bile $\mathrm{pH}$ of $>7.4$ was recently shown to represent appropriate Bicarbonate secretion into bile by Cholangiocytes and therefore protect from ITBL development. The use of different markers implies several critical points, not only the type of marker but also the threshold when to accept or not a graft needs to be defined. Our group has worked on new mitochondrial markers, such as flavoproteins released from mitochondrial complex I during normothermic or hypothermic perfusion. Although metabolic assessment of grafts during machine perfusion appears promising and feasible, validation in clinical trials will be needed before establishing routine perfusate analysis for the difficult decision whether to use or not marginal organs.

The currently used markers include bile bicarbonate, $\mathrm{pH}$ and perfusate lactate clearance, assessed after 2-4 hours of normothermic perfusion $(12,23,48,86)$. However, the declaration that "liver viability assessment is only feasible at normothermic temperatures" should be applied with caution (5). Numerous proteins and metabolites are also released during hypothermic and subnormothermic machine liver perfusion $(90,91)$. A few of them are currently explored to predict liver function and outcomes after transplantation $(19,91,92)$. A similar set of proteins has also been recently described in kidney perfusates and metabolic changes after reperfusion in brain tissue is very comparable to livers (92).

\section{How should future clinical trials be designed?}

The design of the next clinical trial depends on the clinical question to be answered. Here the most burning and difficult one appears as: "Which perfusion technique enables the bighest utilization rate of otherwise discarded livers with the best 


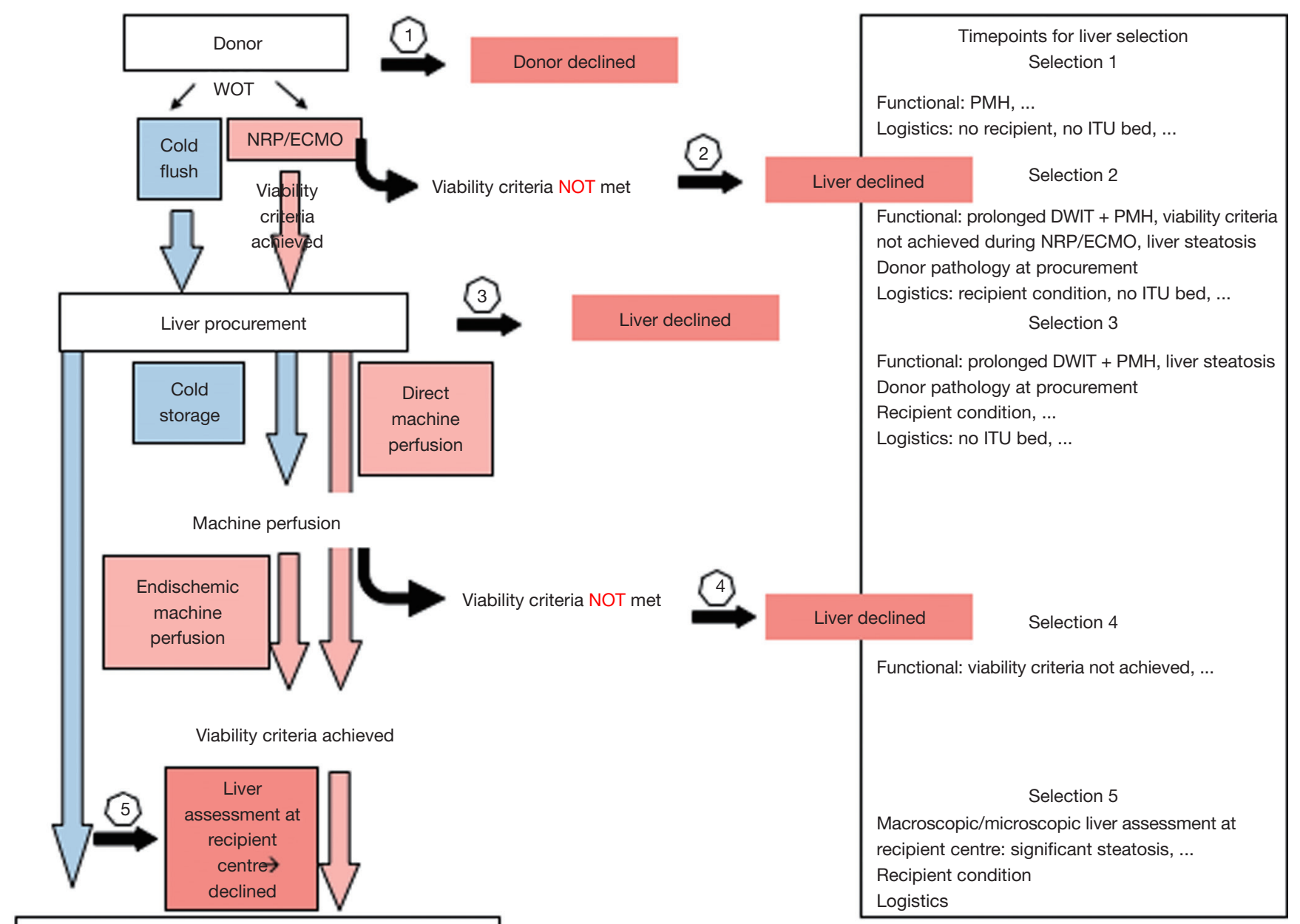

Liver utilized for transplantation

Figure 4 Decision making based on clinical assessment and viability testing in DCD liver transplantation. Throughout the entire process of organ offer, donation, preservation, transport and implantation, decision making is becoming increasingly important and liver grafts are declines at different time points and for various reasons. DCD, donation after circulatory death.

available outcomes, regarding complications, graft- and patient survival". Other important parameters, which require assessment are the reliability of viability markers and how cost effective is a specific preservation approach. While several RCTs are currently recruiting transplant recipients, the next clinically relevant trial should compare most recent concepts. For example, continuous with endischemic NMP compared to endischemic HOPE. However, to find a uniform definition of donor and graft risk with guidelines for clinicians how to utilize higher risk livers best for a certain population of liver recipients is at least equally important.

In the next years, we may therefore expect the field to become even more interesting though possibly more difficult, as new devices, technologies and markers will appear and require further comparisons through clinical trials.

\section{Acknowledgments}

None.

\section{Footnote}

Conflicts of Interest: The authors have no conflicts of interest to declare. 


\section{References}

1. Dutkowski P, De Rougemont O, Clavien PA. Alexis Carrel: Genius, innovator and ideologist. Am J Transplant 2008;8:1998-2003.

2. Detelich D, Markmann JF. The dawn of liver perfusion machines. Curr Opin Organ Transplant 2018;23:151-61.

3. Schlegel A, Kron P, Graf R, et al. Warm vs. cold perfusion techniques to rescue rodent liver grafts. J Hepatol 2014;61:1267-75.

4. Selten J, Schlegel A, de Jonge J, et al. Hypo- and normothermic perfusion of the liver: Which way to go? Best Pract Res Clin Gastroenterol 2017;31:171-9.

5. Boteon YL, Afford S, Mergental H. Pushing the Limits: Machine Preservation of the Liver as a Tool to Recondition High-Risk Grafts. Curr Transplant Rep 2018;5:113-20.

6. Nasralla D, Coussios CC, Mergental H, et al. A randomized trial of normothermic preservation in liver transplantation. Nature 2018;557:50-6.

7. Hosgood SA, Barlow AD, Dormer J, et al. The use of ex-vivo normothermic perfusion for the resuscitation and assessment of human kidneys discarded because of inadequate in situ perfusion. J Transl Med 2015;13:329.

8. Hosgood SA, Van Heurn E, Nicholson ML. Normothermic machine perfusion of the kidney: Better conditioning and repair? Transpl Int 2015;28:657-64.

9. Bruinsma BG, Yeh H, Ozer S, et al. Subnormothermic machine perfusion for ex vivo preservation and recovery of the human liver for transplantation. Am J Transplant 2014;14:1400-9.

10. Bruinsma BG, Berendsen TA, Izamis ML, et al. Determination and extension of the limits to static cold storage using subnormothermic machine perfusion. Int J Artif Organs 2013;36:775-80.

11. Gringeri E, Bonsignore P, Bassi D, et al. Subnormothermic machine perfusion for non-heart-beating donor liver grafts preservation in a swine model: A new strategy to increase the donor pool? Transplant Proc 2012;44:2026-8.

12. Mergental H, Perera MT, Laing RW, et al. Transplantation of Declined Liver Allografts Following Normothermic ExSitu Evaluation. Am J Transplant 2016;16:3235-45.

13. Hessheimer AJ, Coll E, Torres F, et al. Normothermic regional perfusion versus super rapid recovery in controlled donation after circulatory death liver transplantation. J Hepatol 2019;70:658-65.

14. Watson CJ, Hunt F, Messer S, et al. In situ normothermic perfusion of livers in controlled circulatory death donation may prevent ischemic cholangiopathy and improve graft survival. Am J Transplant 2019;19:1745-58.

15. He X, Guo Z, Zhao Q, et al. The first case of ischemiafree organ transplantation in humans: A proof of concept. Am J Transplant 2018;18:737-44.

16. Westerkamp AC, Mahboub P, Meyer SL, et al. Endischemic machine perfusion reduces bile duct injury in donation after circulatory death rat donor livers independent of the machine perfusion temperature. Liver Transpl 2015;21:1300-11.

17. de Meijer VE, Fujiyoshi M, Porte RJ. Ex situ machine perfusion strategies in liver transplantation. J Hepatol 2019;70:203-5.

18. Schlegel A, Muller X, Dutkowski P. Hypothermic Liver Perfusion. Curr Opin Organ Transplant 2017;22:563-70.

19. Kron P, Schlegel A, Mancina L, et al. Hypothermic oxygenated perfusion (HOPE) for fatty liver grafts in rats and humans. J Hepatol 2017. [Epub ahead of print].

20. Watson CJ, Kosmoliaptsis V, Randle LV, et al. Normothermic perfusion in the assessment and preservation of declined livers before transplantation: Hyperoxia and vasoplegia-important lessons from the first 12 cases. Transplantation 2017;101:1084-98.

21. Laing RW, Mergental H, Yap C, et al. Viability testing and transplantation of marginal livers (VITTAL) using normothermic machine perfusion: study protocol for an open-label, non-randomised, prospective, single-arm trial. BMJ Open 2017;7:e017733.

22. Minor T, Efferz P, Fox M, et al. Controlled oxygenated rewarming of cold stored liver grafts by thermally graduated machine perfusion prior to reperfusion. Am J Transplant 2013;13:1450-60.

23. Watson CJ, Jochmans I. From "Gut Feeling” to Objectivity: Machine Preservation of the Liver as a Tool to Assess Organ Viability. Curr Transplant Rep 2018;5:72-81.

24. Schlegel AA, Kalisvaart M, Muiesan P. Machine perfusion in liver transplantation: an essential treatment or just an expensive toy? Minerva Anestesiol 2018;84:236-45.

25. Schlegel A, Rougemont O De, Graf R, et al. Protective mechanisms of end-ischemic cold machine perfusion in DCD liver grafts. J Hepatol 2013;58:278-86.

26. Hosgood SA, Moore T, Kleverlaan T, et al. Haemoadsorption reduces the inflammatory response and improves blood flow during ex vivo renal perfusion in an experimental model. J Transl Med 2017;15:216.

27. Chouchani ET, Pell VR, James AM, et al. A Unifying Mechanism for Mitochondrial Superoxide Production during Ischemia-Reperfusion Injury. Cell Metab 2016;23:254-63. 
28. van Golen RF, van Gulik TM, Heger M. Mechanistic overview of reactive species-induced degradation of the endothelial glycocalyx during hepatic ischemia/reperfusion injury. Free Radic Biol Med 2012;52:1382-402.

29. Stegemann J, Minor T. Energy charge restoration, mitochondrial protection and reversal of preservation induced liver injury by hypothermic oxygenation prior to reperfusion. Cryobiology 2009;58:331-6.

30. Michel SG, La Muraglia GM, Madariaga ML, et al. Twelve-hour hypothermic machine perfusion for donor heart preservation leads to improved ultrastructural characteristics compared to conventional cold storage. Ann Transplant 2015;20:461-8.

31. Chouchani ET, Pell VR, Gaude E, et al. Ischaemic accumulation of succinate controls reperfusion injury through mitochondrial ROS. Nature 2014;515:431-5.

32. Mills EL, Kelly B, Logan A, et al. Succinate Dehydrogenase Supports Metabolic Repurposing of Mitochondria to Drive Inflammatory Macrophages. Cell 2016;167:457-70.e13.

33. Siebels I, Dröse S. Q-site inhibitor induced ROS production of mitochondrial complex II is attenuated by TCA cycle dicarboxylates. Biochim Biophys Acta 2013;1827:1156-64.

34. Takakuwa $Y$, Nishino $H$, Ishibe $Y$, et al. Properties and kinetics of membrane-bound enzymes when both the enzyme and substrate are components of the same microsomal membrane. Studies on lathosterol 5-desaturase. J Biol Chem 1994;269:27889-93.

35. Murphy MP. How mitochondria produce reactive oxygen species. Biochem J 2009;417:1-13.

36. Dutkowski P, Clavien P. Uploading cellular batteries: Caring for mitochondria is key. Liver Transpl 2018;24:462-4.

37. Land WG. Emerging role of innate immunity in organ transplantation. Part I: Evolution of innate immunity and oxidative allograft injury. Transplant Rev (Orlando) 2012;26:60-72.

38. Kron P, Schlegel A, de Rougemont O, Oberkofler CE, et al. Short, Cool, and Well Oxygenated - HOPE for Kidney Transplantation in a Rodent Model. Ann Surg 2016;264:815-22.

39. Hashimoto K, Cypel M, Kim H, et al. Soluble Adhesion Molecules During Ex Vivo Lung Perfusion Are Associated With Posttransplant Primary Graft Dysfunction. Am J Transplant 2017;17:1396-404.

40. Iskender I, Cosgun T, Arni S, et al. Cytokine filtration modulates pulmonary metabolism and edema formation during ex vivo lung perfusion [Abstract]. J Hear Lung
Transpl 2016;35:S142-3.

41. Boteon YL, Laing RW, Schlegel A, et al. Combined Hypothermic and Normothermic Machine Perfusion Improves Functional Recovery of Extended Criteria Donor Livers. Liver Transpl 2018;24:1699-715.

42. Abele D, Heise K, Pörtner HO, et al. Temperaturedependence of mitochondrial function and production of reactive oxygen species in the intertidal mud clam Mya Mya arenaria. J Exp Biol 2002;205:1831-41.

43. Abele D. Toxic oxygen: The radical life-giver. Nature 2002;420:27.

44. Dufour S, Rousse N, Canioni P, et al. Top-down control analysis of temperature effect on oxidative phosphorylation. Biochem J 1996;314:743-51.

45. Dugbartey GJ, Hardenberg MC, Kok WF, et al. Renal Mitochondrial Response to Low Temperature in NonHibernating and Hibernating Species. Antioxid Redox Signal 2017;27:599-617.

46. MacDonald JA, Storey KB. cAMP-dependent protein kinase from brown adipose tissue: temperature effects on kinetic properties and enzyme role in hibernating ground squirrels. J Comp Physiol B 1998;168:513-25.

47. Burlage LC, Hessels L, van Rijn R, et al. Opposite acute potassium and sodium shifts during transplantation of hypothermic machine perfused donor livers. Am J Transplant 2019;19:1061-71.

48. Westerkamp AC, Karimian N, Matton A, et al. Oxygenated Hypothermic Machine Perfusion After Static Cold Storage Improves Hepatobiliary Function of Extended Criteria Donor Livers. Transplantation 2016;100:825-35.

49. Blier PU, Abele D, Munro D, et al. What modulates animal longevity? Fast and slow aging in bivalves as a model for the study of lifespan. Semin Cell Dev Biol 2017;70:130-40.

50. Wang CH, Wu SB, Wu YT, et al. Oxidative stress response elicited by mitochondrial dysfunction: Implication in the pathophysiology of aging. Exp Biol Med (Maywood) 2013;238:450-60.

51. Karki R, Man SM, Kanneganti TD. Inflammasomes and Cancer. Cancer Immunol Res 2017;5:94-9.

52. Schlegel A, Muller X, Dutkowski P. Hypothermic Machine Preservation of the Liver: State of the Art. Curr Transplant Rep 2018;5:93-102.

53. Schlegel A, Muller X, Kalisvaart M, et al. Outcomes of liver transplantations from donation after circulatory death (DCD) treated by hypothermic oxygenated perfusion (HOPE) before implantation. J Hepatol 2019;70:50-7.

54. van Rijn R, Karimian N, Matton AP, et al. Dual 
hypothermic oxygenated machine perfusion in liver transplants donated after circulatory death. Br J Surg 2017;104:907-17.

55. De Carlis L, De Carlis R, Lauterio A, et al. Sequential Use of Normothermic Regional Perfusion and Hypothermic Machine Perfusion in Donation After Cardiac Death Liver Transplantation With Extended Warm Ischemia Time. Transplantation 2016;100:e101-2.

56. De Carlis R, De Carlis R, Lauterio A, et al, Successful donation after cardiac death liver transplants with prolonged warm ischemia time using normothermic regional perfusion. Liver Transpl 2017;23:166-73.

57. Muller X, Marcon F, Sapisochin G, et al. Defining Benchmarks in Liver Transplantation: A Multicenter Outcome Analysis Determining Best Achievable Results. Ann Surg 2018;267:419-25.

58. Marcon F, Schlegel AA, Bartlett D, et al. Utilisation of declined liver grafts yields comparable transplant outcomes and previous decline should not be a deterrent to graft use. Transplantation 2018. [Epub ahead of print].

59. European Association for the Study of the Liver.EASL Clinical Practice Guidelines: Liver transplantation. J Hepatol 2016;64:433-85.

60. Kalisvaart M, de Haan JE, Polak WG, et al. Onset of Donor Warm Ischemia Time in Donation After Circulatory Death Liver Transplantation: Hypotension or Hypoxia? Liver Transpl 2018;24:1001-10.

61. Hessheimer A, Coll E, Valdivieso A, et al. Superior outcomes using normothermic regional perfusion in cDCD liver transplantation. Available online: https:// atcmeetingabstracts.com/abstract/superior-outcomesusing-normothermic-regional-perfusion-in-cdcd-livertransplantation/

62. Schlegel A, Kalisvaart M, Scalera I, et al. The UK DCD Risk Score: A new proposal to define futility in donationafter-circulatory-death liver transplantation. J Hepatol 2018;68:456-64.

63. Goldberg DS, Karp SJ, McCauley ME, et al. Interpreting Outcomes in DCDD Liver Transplantation: First Report of the Multicenter IDOL Consortium. Transplantation 2017;101:1067-73.

64. Croome KP, Mathur AK, Lee DD, et al. Outcomes of Donation after Circulatory Death Liver Grafts from Donors 50 Years or Older: A Multicenter Analysis. Transplantation 2018;102:1108-14.

65. Schlegel A, Scalera I, Perera M, et al. Impact of donor age in donation after cardiac death liver transplantation: Is the cut-off " 60 " still of relevance? Liver Transpl
2018;24:352-62.

66. Schlegel A, Muller X, Kalisvaart M, et al. Outcomes of liver transplantations from donation after circulatory death (DCD) treated by hypothermic oxygenated perfusion (HOPE) before implantation. J Hepatol 2019;70:50-7.

67. Dutkowski P, Oberkofler CE, Slankamenac K, et al. Are there better guidelines for allocation in liver transplantation? A novel score targeting justice and utility in the model for end-stage liver disease era. Ann Surg 2011;254:745-53; discussion 753.

68. Graham JA, Guarrera JV. "resuscitation" of marginal liver allografts for transplantation with machine perfusion technology. J Hepatol 2014;61:418-31.

69. Bruzzone P, Giannarelli D, Adam R. A preliminary european liver and intestine transplant associationeuropean liver transplant registry study on informed recipient consent and extended criteria liver donation. Transplant Proc 2013;45:2613-5.

70. Nemes B, Gámán G, Polak WG, et al. Extended criteria donors in liver transplantation Part I: reviewing the impact of determining factors. Expert Rev Gastroenterol Hepatol 2016;10:827-39.

71. Bertuzzo VR, Cescon M, Odaldi F, et al. Actual Risk of Using Very Aged Donors for Unselected Liver Transplant Candidates. Ann Surg 2017;265:388-96.

72. Collett D, Friend PJ, Watson CJ. Factors Associated With Short- and Long-term Liver Graft Survival in the United Kingdom: Development of a UK Donor Liver Index. Transplantation 2017;101:786-92.

73. Khorsandi SE, Giorgakis E, Vilca-Melendez H, et al. Developing a donation after cardiac death risk index for adult and pediatric liver transplantation. World J Transplant 2017;7:203-12.

74. Briceño J, Ciria R, De La Mata M. Donor-recipient matching: Myths and realities. J Hepatol 2013;58:811-20.

75. Schlegel A, Kalisvaart M, Isaac JR, et al. Reply to: "DCD consensus and futility in liver transplantation". J Hepatol 2018;69:257-8.

76. Muller X, Schlegel A, Würdinger M, et al. Can hypothermic oxygenated perfusion (HOPE) rescue futile DCD liver grafts? HPB (Oxford) 2019. [Epub ahead of print].

77. Hoyer DP, Mathé Z, Gallinat A, et al. Controlled Oxygenated Rewarming of Cold Stored Livers Prior to Transplantation: First Clinical Application of a New Concept. Transplantation 2016;100:147-52.

78. Bernardi M, Gitto S, Biselli M. The MELD score in patients awaiting liver transplant: Strengths and weaknesses. J Hepatol 2011;54:1297-306. 
79. Nagai S, Yoshida A, Facciuto M, et al. Ischemia time impacts recurrence of hepatocellular carcinoma after liver transplantation. Hepatology 2015;61:895-904.

80. Kornberg A, Witt U, Kornberg J, et al. Extended Ischemia Times Promote Risk of HCC Recurrence in Liver Transplant Patients. Dig Dis Sci 2015;60:2832-9.

81. Orci LA, Lacotte S, Delaune V, et al. Effects of the gutliver axis on ischaemia-mediated hepatocellular carcinoma recurrence in the mouse liver. J Hepatol 2018;68:978-85.

82. Lim C, Azoulay D. Ischemia-Reperfusion Injury and Regrowth of Liver Cancers: To Clamp or Not to Clamp? Dig Dis Sci 2014;59:2030-1.

83. Croome KP, Lee DD, Burns JM, et al. The Use of Donation After Cardiac Death Allografts Does Not Increase Recurrence of Hepatocellular Carcinoma. Am J Transplant 2015;15:2704-11.

84. Khorsandi SE, Yip VS, Cortes M, et al. Does Donation After Cardiac Death Utilization Adversely Affect Hepatocellular Cancer Survival? Transplantation 2016;100:1916-24.

85. Jochmans I, Akhtar MZ, Nasralla D, et al. Past, Present, and Future of Dynamic Kidney and Liver Preservation and Resuscitation. Am J Transplant 2016;16:2545-55.

86. Laing RW, Mergental H, Yap C, et al, Viability testing and transplantation of marginal livers (VITTAL) using

Cite this article as: Schlegel A, Muller X, Dutkowski P. Machine perfusion strategies in liver transplantation. HepatoBiliary Surg Nutr 2019;8(5):490-501. doi: 10.21037/ hbsn.2019.04.04 normothermic machine perfusion: study protocol for an open-label, non-randomised, prospective, single-arm trial. BMJ Open 2017;7:e017733.

87. Saito T, Takahashi H, Kaneda H, et al. Impact of cytokine expression in the pre-implanted donor lung on the development of chronic lung allograft dysfunction subtypes. Am J Transplant 2013;13:3192-201.

88. Ceresa C, Nasralla D, Watson C, et al. The effect of normothermic machine perfusion after cold storage in liver transplantation: a multicentre prospective clinical trial. ILTS 2018;102:27.

89. Raptis DA, Fischer MA, Graf R, et al. MRI: The new reference standard in quantifying hepatic steatosis? Gut 2012;61:117-27.

90. Faitot F, Besch C, Battini S, et al. Impact of real-time metabolomics in liver transplantation: Graft evaluation and donor-recipient matching. J Hepatol 2018;68:699-706.

91. Bruinsma BG, Sridharan GV, Weeder PD, et al. Metabolic profiling during ex vivo machine perfusion of the human liver. Sci Rep 2016;6:22415.

92. Kim M, Stepanova A, Niatsetskaya Z, et al. Attenuation of oxidative damage by targeting mitochondrial complex I in neonatal hypoxic-ischemic brain injury. Free Radic Biol Med 2018;124:517-24. 